\title{
Vorbemerkungen der Herausgeber zum Themenheft "Simulation für betriebswirtschaftliche Entscheidungsprozesse“
}

In der Zeit vom 6.-8. März 1989 fand in Braunlage das 2. Symposium „Simulation als betriebliche Entscheidungshilfe " unter unserer wissenschaftlichen und organisatorischen Leitung statt. Aufgrund der Bedeutung der Simulation für die betriebliche Entscheidungsfindung und der positiven Resonanz auf die Tagung entschloß sich der DGOR-Vorstand, diese Veranstaltung zum Anlaß zu nehmen, ein Sonderheft des ORSpektrums zum Thema „Simulation für betriebswirtschaftliche Entscheidungsprozesse" herauszugeben. Dabei sollte es sich nicht um eine Wiedergabe der Vorträge handeln, sondern es sollte vielmehr dargestellt werden, in welchen betriebswirtschaftlichen Bereichen der Simulation heute gearbeitet wird. Da die wesentlichen Tagungsbeiträge des Symposiums bereits Eingang in den Band II "Simulation als betriebliche Entscheidungshilfe" gefunden hatten und dieses Sonderheft laut Vorgabe auch kein Tagungsband sein soll, entschlossen wir uns, - ohne Anspruch auf Vollständigkeit $^{1}$ - Beiträge über die ganze Breite der Simulation von namhaften Fachvertretern und Anwendern anzuwerben, die sich in letzter Zeit besonders intensiv mit der Simulation befaßt hatten. Dabei wurden bewußt drei Problembereiche verfolgt:

a) Simulationskonzepte und -werkzeuge im Wandel

b) Simulationsanwendungen für die Praxis

c) Simulationsanwendungen bei Planspielen

Die Beiträge, die sich hierfür anbieten, sollen nun kurz eingeordnet werden.

In den angesprochenen Bereichen macht sich eine Verschiebung der Simulation in Richtung auf die Bewältigung komplexerer Probleme auf der Basis von effektiveren Tools bemerkbar. Dieses hat einen erheblichen Einfluß auf die Modellierung. Dieser Problematik wird der erste Beitrag gewidmet.

\footnotetext{
${ }^{1}$ Dieser ist allein aufgrund des Umfanges eines solchen Heftes unmöglich.
}

Auch die Weiterentwicklung der Tools, die ihre Vorläufer in SIMULA und GPSS hatten, führt immer mehr in vollständigere Software-Simulationsumgebungen, wobei Aspekte der Visualisierung zunehmend berücksichtigt werden. Dieses wird an der Konzeption von ProfiSEE von Mosner/Heeg deutlich, und auch der Beitrag von Vaessen zeigt, wie sehr diese Werkzeuge und damit derartige Konzepte für die betriebliche Anwendung benötigt werden.

Der mehr grundsätzlichen Frage, wie sich Simulationsmodelle und Expertensysteme gegenseitig unterstützen können, widmet sich Schmidt in seinem Beitrag.

Bei den Arbeiten von Bloech, Mertens/Ringlstetter und Hummeltenberg/Pressmar handelt es sich eher um anwendungsorientierte Konzepte, wobei Bloech seinen Schwerpunkt in der Einsatzsteuerung des Personals im Materialeinkauf sieht, während bei Mertens/Ringlstetter die CIM-Planung auf der Basis von Expertensystemen im Vordergund steht.

Hummeltenberg/Pressmar zeigen dagegen anhand von Beispielen, daß sowohl die Simulation als auch die Verfahren mathematischer Optimierung ihre Daseinsberechtigung im Bereich der Produktions- und Ablaufplanung haben, daß man aber auch die Grenzen der Verfahren sehr genau kennen muß. Insofern liegt hier nach der Vorgabe eines Anwendungsfeldes der Schwerpunkt auf dem Vergleich.

Im Gegensatz zur letzten Gruppe der Beiträge sind die von Stähly und von Vaessen eindeutig und ausschließlich der Anwendung zuzuordnen. Dabei zeigt Vaessen, wie man unter Einsatz der entsprechenden Tools in kurzer Zeit ein visuelles, interaktives Simulationssystem für die chemische Industrie erhalten konnte. Stähly dagegen entwickelt auf Basis der klassischen Simulationssprache SIMULA ein ausgesprochen komplexes System für die Einsatzplanung in Katastrophenfällen anhand realer Daten. Dabei werden aber auch die Grenzen der heutigen Modellierung deutlich und 
die Tatsache, daß demnächst der Schritt zur Parallelisierung getan werden sollte.

Der letzte Schwerpunkt gilt der Simulation in Planspielen, weil dadurch die Modellierung der Entscheidenden und ihrer Entscheidungen für die Ausbildung zu guten Managern erfolgt. Dieser Fragestellung widmen sich Schiemenz und Thome, wobei Schiemenz für das Planspiel TOPIC ein Simulationssystem EUSTOPIC zur Entscheidungsunterstützung in vielen Bereichen entwickelt hat. Das von Thome dargestellte Spiel VULCAN dagegen beschreibt ein Ausbildungssystem, in dem mehrere Unternehmen durch verschiedene Universitäten simuliert werden und dabei die
Lösungen der Bürokommunikation über das deutsche Forschungsnetz (DFN) einsetzen.

Die Herausgeber hoffen, daß es ihnen mit der Breite dieser Arbeiten gelungen ist, einen - wenn auch nicht vollständigen, so doch weitgehend repräsentativen Querschnitt über die Simulation und ihre Anwendung gegeben zu haben. Gleichzeitig hoffen wir, daß dieses Heft Anlaß gibt, weitere zu jeweils einem abgegrenzten Themengebiet folgen zu lassen.

J. Biethahn

W. Hummeltenberg

B. Schmidt

\section{Vorwort des Hauptherausgebers}

Mit dem vorliegenden Heft erscheint zum ersten Mal ein OR-SPEKTRUM als „Special Issue“ zu einem abgegrenzten Themenbereich. Die Idee, dieses von anderen OR-Zeitschriften schon seit längerem praktizierte Konzept auch für unsere Zeitschrift einmal zu übernehmen, war insbesondere darin begründet, dem von der Leserschaft und den DGOR-Mitgliedern geäuBerten Wunsch nach einem insgesamt größeren Praxisbezug der ORS-Beiträge nachzukommen.

Die Herausgeber des OR-SPEKTRUM hoffen, daß mit dem vorliegenden Themenheft „Simulation für betriebswirtschaftliche Entscheidungsprozesse“ dieses Ziel erreicht wird und daß sowohl dieses erste Sonderheft als auch das Konzept der „Special Issues“ insgesamt bei der Leserschaft ankommen. Für diesen Fall ist die Herausgabe weiterer Sonderhefte zu Themenbereichen von allgemeinem, breitem Interesse in losen Abständen und jeweils unter der Federführung eines Gastherausgebers vorgesehen. Dabei soll jedoch für das ORS der Charakter einer wissenschaftlichen Zeitschrift, der insbesondere durch thematisch eigenständige, aktuelle Beiträge geprägt ist, grundsätzlich beibehalten werden.

Es war ein gutes Zusammentreffen, daß gleichzeitig mit der Idee der Herausgabe von „Special Issues“, sich mit dem Symposium in Braunlage sowohl eine attraktive Thematik als auch mit den Organisatoren dieser
Tagung dem Projekt „Special Issue“ zugetane, kompetente Gastherausgeber anboten. $\mathrm{Da} ß$ es von der Idee bis zur Druckrealisierung kaum mehr als ein halbes Jahr bedurfte, ist insbesondere der Bereitschaft der Autoren und dem Engagement der Gastherausgeber zu verdanken. Insbesondere möchte ich an dieser Stelle Herrn Biethahn meinen Dank aussprechen, der die gesamte Koordination in so vorzüglicher Weise übernommen hat.

Angesichts der Bedeutung des Sonderheftes und dem erwarteten Umfang der Beiträge Rechnung tragend, hatten wir - wie sicher allgemein nicht unbemerkt geblieben ist - bei den ersten Heften des 11. Jahrgangs jeweils eine bestimmte Anzahl von Seiten für das Sonderheft eingespart. Der Gesamtumfang der zehn Beiträge übersteigt jedoch trotzdem den für das vierte Heft zur Verfügung stehenden Raum. Aus produktionstechnischen Gründen ist es damit leider notwendig geworden, die Beiträge des dritten Themenbereichs "Simulationsanwendungen bei Planspielen“ aus diesem vierten Heft auszugliedern. Die beiden Fachbeiträge von Schiemenz und Thome werden daher im ersten Heft des zwölften Jahrgangs in einer speziellen Rubrik nachträglich erscheinen.

U. Derigs $\quad$ Bayreuth, November 1989 